\title{
Age structure, environmental fluctuations, and hermaphroditic sex allocation
}

\author{
Shripad Tuljapurkar
}

Biological Sciences, Stanford University, Stanford, CA 94305, U.S.A.

This paper studies sex allocation in an age-structured population of hermaphrodites living in a temporally fluctuating environment. The general condition for the evolutionary stable state (ESS) of allocation is derived for densityindependent dynamics. This condition is used to determine the effect on the deterministic ESS of a dependence of survival rates on allocation. It is also used to identify the special conditions under which a stochastic ESS is given by a product rule and show how demographic structure and the correlation structure of vital rates determines the stochastic ESS.

\section{INTRODUCTION}

Charnov (1982) defines sex allocation to include the evolution of sex ratio in dioecious species, sex change in sequential hermaphrodites, and the allocation of resources to male versus female function in simultaneous hermaphrodites. Given simplifying assumptions about population structure, a phenotypic ESS (evolutionarily stable state: Maynard Smith 1982) in each of these problems obeys a general optimality principle derived by Charnov $(1979,1982)$. The addition of realistic features of population structure and environment can lead to very different types of ESS. Werren and Charnov (1978) and Seger (1983) demonstrated theoretically the effect of changing age structure on sex ratio. Bierzychudek (1984) demonstrated experimentally the joint effect of age structure and temporal variability on sex change in a sequential hermaphrodite. Charnov (1988) studied the ESS for sex allocation in a hermaphrodite using simple models of overlapping generations, and initiated a study of the effects of temporal variability.

This paper considers sex allocation in an agestructured, density-independent population of simultaneous hermaphrodites in a temporally variable environment. I describe temporal variation by taking population vital rates to be random stationary time-series. My definition of an ESS, as in Charnov (1979), Charlesworth (1980) and Tuljapurkar (1982), is based on analysis of the dynamics of rare alleles at a single diploid locus. The main results are:

(1) Derivation of the general criterion which defines an evolutionarily stable (ES) sex allocation.

(2) A special case of the above criterion describes age-structured populations in a constant environment. I use it to show how the ESS shifts when survival rates depend on sex allocation.

(3) Identification of conditions under which the criterion of (1) reduces to Charnov's (1986, 1988) production-maximization rule. The key assumptions are that the shape of the female production function does not vary with age or over time, and the shape of the male production function is age-independent and uncorrelated with all other time-varying quantities.

(4) An example is used to show how age and uncertainty interact to determine the ESS when the special conditions in (3) are not met.

\section{ESS WITH AGE STRUCTURE AND TEMPORAL VARIATION}

I follow Charnov $(1982,1988)$ and consider a large hermaphroditic plant population. There are $k$ discrete age classes (seeds are age class 1 ) and mating is random. Initially all individuals are homozygous 
for allele $A$ at the locus which controls allocation between pollen and seeds. Define:

$r$, the fraction of resources allocated at every age to pollen (versus seeds);

$F_{i}(r, t)$, the per capita seed set in age-class $i$ from time $t-1$ to $t$;

$M_{i}(r, t)$, the per capita pollen produced in ageclass $i$ from $t-1$ to $t$;

$S_{i}(r, t)$, the survival rate of age-class $i$ individuals from time $t-1$ to $t$;

$N_{i}(t)$, the number of age-class $i$ individuals at time $t$.

The dynamics of this population of $A A^{\prime}$ 's follow the equation

$$
\begin{array}{r}
\left(\begin{array}{c}
N_{1}(t) \\
N_{2}(t) \\
\vdots \\
N_{k}(t)
\end{array}\right)= \\
\left(\begin{array}{ccc}
F_{1}(r, t) & F_{2}(r, t) & F_{3}(r, t) \\
S_{i}(r, t) & 0 & 0 \\
\vdots & \vdots & \vdots \\
0 & 0 & 0 \\
& \ldots & F_{k}(r, t) \\
& \ldots & 0 \\
& \ldots & \vdots \\
S_{k-1}(r, t) & 0
\end{array}\right)\left(\begin{array}{c}
N_{1}(t-1) \\
N_{2}(t-1) \\
\vdots \\
N_{k}(t-1)
\end{array}\right) .
\end{array}
$$

The time-dependence of vital rates in (1) derives from a randomly varying environment. In a real plant population, age-class 1 will be seeds and so $F_{1}=0$. However, it is convenient to do the analysis with $F_{1}$ present, especially for comparison with simple non-age-structured models. I assume that

(a) the vital rates form a stationary stochastic process,

(b) the moments of the vital rates are bounded,

(c) the stochastic process has a rapidly disappearing memory of its past,

(d) demographic weak ergodicity holds for the random rates.

More general assumptions can be made; see, e.g. Heyde and Cohen (1985) without changing what follows. With assumptions (a)-(d) the dynamics of (1) have two particularly important features. First, the population growth rate is a nonrandom number

$$
a=\lim _{t \rightarrow \infty} \frac{1}{t} \log \left[\sum_{i} N_{i}(t)\right] .
$$

Second, the population age-structure (the ratios $\left.Y_{i}(t)=N_{i}(t) /\left[\sum N_{i}(t)\right]\right)$ converges to a random time-dependent sequence independent of the initial population.

Consider now the fate of a rare allele $B$ which causes heterozygotes $A B$ to allocate a fraction $r_{1}$ of resources to pollen. Initially allele $B$ is carried mainly by heterozygotes. Let

$$
\varepsilon(t)=\left(\varepsilon_{1}(t), \varepsilon_{2}(t), \ldots, \varepsilon_{k}(t)\right)
$$

be the age-vector of heterozygote population numbers. I now obtain an equation which describes changes when $\varepsilon$ is small (as in Charnov, 1979; Tuljapurkar, 1982). Seeds produced by $A B$ 's will, to a good approximation, be fertilized by $A A$ pollen so half of these seeds will be $A B$. Pollen from $A B$ 's will compete with $A A$ pollen to fertilize mostly $A A$ seeds; half of the successful fertilizations so produced will be $A B$. Hence the number of $A B$ seeds changes as

$$
\begin{aligned}
\varepsilon_{1}(t)= & \frac{1}{2} \sum F_{i}\left(r_{1}, t\right) \varepsilon_{i}(t-1) \\
& +\frac{\left[\sum F_{i}(r, t) N_{i}(t-1)\right]}{2\left[\sum M_{i}(r, t) N_{i}(t-1)\right]} \sum M_{i}\left(r_{1}, t\right) \varepsilon_{i}(t-1), \\
= & \sum \Psi_{i}(t) \varepsilon_{i}(t-1), \text { say. }
\end{aligned}
$$

On the right side of the first line above, divide numerator and denominator by the total number of $A A$ to find

$$
\begin{aligned}
\Psi_{i}(t)= & \frac{1}{2} F_{i}\left(r_{1}, t\right) \\
& +\frac{\left[\sum F_{i}(r, t) Y_{i}(t-1)\right]}{2\left[\sum M_{i}(r, t) Y_{i}(t-1)\right]} M_{i}\left(r_{1}, t\right) .
\end{aligned}
$$

Here $Y_{i}(t-1)$ is the proportion of $A A$ in age-class $i$ at time $t-1$ in the homozygous population of equation (1). Thus heterozygote numbers follow the linear equation

$$
\varepsilon(t+1)=\left(\begin{array}{cccc}
\Psi_{1}(t) & \Psi_{2}(t) & \ldots & \Psi_{k}(t) \\
S_{1}(r, t) & 0 & \ldots & 0 \\
\vdots & \vdots & \ldots & \vdots \\
0 & \vdots & S_{k-1}(r, t) & 0
\end{array}\right) \varepsilon(t) .
$$

The sequence of random matrices in this equation yields a (nonrandom) growth rate $a_{1}$, which depends on both $r$ and $r_{1}$. Allele $B$ invades (i.e., increases in frequency) if

$$
a_{1}>a \text {. }
$$

The condition for an ESS is

$$
\left.\frac{\partial}{\partial r_{1}} a_{1}\left(r_{1}, r\right)\right|_{r_{1}=r}=0 .
$$

Readers unfamiliar with the stochastic growth rate $a$ should think of it as the exponential growth rate resulting from the application of a long series of matrices with the appropriate random elements. (See Tuljapurkar and Orzack, 1980; Cohen, 1979; 
Tuljapurkar, 1989 for further details on random demography).

\section{STOCHASTIC RULE FOR ESS ALLOCATION}

Condition (5) is general but not directly useable; I will now translate it into an interpretable form. In equation ( 1$)$, denote the (random) matrix by $\boldsymbol{X}(t)$. The sequence $\boldsymbol{X}(1), \boldsymbol{X}(2), \boldsymbol{X}(3)$, etc., is a stationary random process, and it determines a time-dependent random set of age-structure vectors $\boldsymbol{U}(t)$ and reproductive value vectors $\boldsymbol{V}(t)$, such that the product

$$
(\boldsymbol{X}(t) \boldsymbol{X}(t-1) \cdots \boldsymbol{X}(1)) \sim \rho_{t} \frac{\boldsymbol{U}(t) \boldsymbol{V}^{T}(t)}{\left(\boldsymbol{V}^{T}(t) \boldsymbol{U}(t)\right)} .
$$

Here $T$ indicates a transpose, $\rho_{t}$ is the growth rate over $t$ generations, and the symbol " $\sim$ " means that the two sides are asymptotically equal as $t \rightarrow \infty$. We can compute $\boldsymbol{U}(t), \boldsymbol{V}(t)$ as the right, left eigenvectors for the dominant eigenvalue of the product matrix in (6). The age-structure of the resident $A A$ homozygotes in (1), given by $\boldsymbol{Y}(t)$, converges to $\boldsymbol{U}(t)$ as $t$ increases, independent of the initial stage. The one-period growth rate of $A A$ 's at time $t$ is given by

$$
\lambda(t+1)=\sum_{i}(\boldsymbol{X}(t+1) \boldsymbol{U}(t))_{i}
$$

In the Appendix, I show that (5) reduces to the condition

$$
E\left\{\frac{\boldsymbol{V}^{T}(t) \Delta(t) \boldsymbol{U}(t-1)}{\lambda(t)\left(\boldsymbol{V}^{T}(t) \boldsymbol{U}(t)\right)}\right\}=0,
$$

with

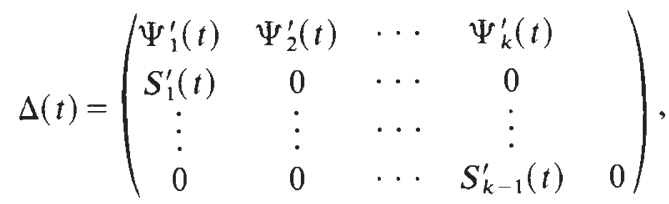

$$
\begin{aligned}
& \Psi_{i}^{\prime}(t)=\frac{1}{2} \bar{F}(r, t)\left[\frac{1}{\bar{F}(r, t)} \frac{\partial F_{i}}{\partial r}+\frac{1}{\bar{M}(r, t)} \frac{\partial M_{i}}{\partial r}\right] \text {, } \\
& \bar{F}(r, t)=\sum_{i} F_{i}(r, t) U_{i}(t-1), \\
& \bar{M}(r, t)=\sum M_{i}(r, t) U_{i}(t-1), \\
& S_{i}^{\prime}(t)=\frac{\partial S_{i}(r, t)}{\partial r} .
\end{aligned}
$$

I refer to (8) as the Rule for Stochastic Evolutionary Stability.
This stochastic rule is best understood by examining special cases in which old results are recovered and new ones uncovered.

\section{SPECIAL CASES}

\section{(i) Fluctuating environment, no age structure}

This is the case studied by Bull (1981) and Charnov (1986). In my formulation, the absence of agestructure means that only $F_{1}(t) \neq 0$ in (1) and only $\Psi_{1}(t) \neq 0$ in (2). The vectors in (8) reduce to $\boldsymbol{U}(t)=\boldsymbol{V}(t) \equiv 1$ while $\lambda(t)=F_{1}(t)$, and $\bar{F}=F_{1}$, $\bar{M}=M_{1}$. The general stochastic rule (8) reduces to Charnov's $(1986,1988)$ product rule

$$
E\left[\frac{1}{F_{1}} \frac{\partial F_{1}}{\partial r}+\frac{1}{M_{1}} \frac{\partial M_{1}}{\partial r}\right]=0 .
$$

The term "product rule" derives from the equivalence of (14) to the maximization of $E \log \left(M_{1} F_{1}\right)$.

\section{(ii) Age structure, constant environment}

Here the random quantities in (1) and (3) are fixed, and $a$ in (2) is just the usual Malthusian parameter $\log \lambda_{0}$. Defining survivorships

$$
l_{1}=1, \quad l_{2}=S_{1}, \quad l_{3}=S_{2} S_{1}, \quad \text { etc., }
$$

we have the characteristic equation

$$
\sum \lambda_{0}^{-i} l_{i} F_{i}=1 \text {. }
$$

The age-vectors $U(t)$ in (8) equal the stable agedistribution $\boldsymbol{U}$ with components

$$
U_{i} \propto l_{i} \lambda_{0}^{-i+1} .
$$

The vectors $\boldsymbol{V}(t)$ in (8) equal the reproductive value vector $\boldsymbol{V}$, whose components are known (e.g., Caswell, 1978) to be

$$
V_{i} \propto \frac{1}{l_{i}}\left[\sum_{j \geq i} F_{j} l_{j} \lambda_{0}^{i-j-1}\right] .
$$

Using these relationships (8) reduces to

$$
\frac{1}{2}\left[\frac{\left\langle\partial F_{i}\right\rangle}{\left\langle F_{i}\right\rangle}+\frac{\left\langle\partial M_{i}\right\rangle}{\left\langle M_{i}\right\rangle}\right]+\sum_{i=1}^{k-1} g_{i}\left(\partial S_{i}\right)=0 .
$$

Here

$$
\begin{aligned}
\left\langle\text { object }_{i}\right\rangle & =\sum_{i=1}^{k} \lambda_{0}^{-i} l_{i}(\text { object })_{i}, \\
\partial(\text { object }) & =\frac{\partial(\text { object })}{\partial r},
\end{aligned}
$$


and

$$
g_{i}=\sum_{j=i+1}^{k} \lambda_{0}^{-j} l_{j} F_{j} .
$$

In the case where survival rates $S_{i}$ do not depend on $r$, (19) reduces to a condition analogous to that of Charnov (1979) for a similar but different allocation problem.

\section{(iii) Survival rate changing with $r$ in a constant environment}

When survival varies with sex allocation, the ESS for $r$ can be computed from (19); this is a new result. I expect survival rates to depend on $r$, because as $r$ increases, the production of females decreases relative to males. If female offspring are more expensive to produce than males, and an individual's energy resources are divided between reproduction and maintenance, adult survival should increase with $r$.

The main effect of survival rate on the ESS is revealed by a simple example, based on Charnov (1988). Consider male and female allocation functions which have the forms

$$
\begin{aligned}
M_{i}(r) & =\alpha_{i} Z r^{g}, \\
F_{i}(r) & =\beta_{i} W(1-r)^{h} .
\end{aligned}
$$

Here we separate the production levels (measured by $Z, W$ ) from the shapes of the production functions (measured by $g, h$ ). Age dependence is contained in $\alpha_{i}$ and $\beta_{i}$. Assume also that

$$
S_{i}(r)=S(r) \text { for all age-classes } i \text {. }
$$

The stochastic rule (19) now reduces to

$$
\frac{g}{r}=\frac{h}{1-r}-2\left(T_{0}-1\right) S^{\prime}(r),
$$

where $S^{\prime}=(\mathrm{d} S / \mathrm{d} r)$ and the mean length of generation is

$$
T_{0}=\sum_{1}^{k} i l_{i} F_{i} \lambda_{0}^{-i}>1
$$

In the cases analyzed by Charnov $(1982,1988)$, $S^{\prime}=0$. If $S^{\prime}>0$ (i.e., survival rate goes up when more male than female orispring are produced), the ES value of $r$ is greater than when $S^{\prime}=0$. Fig. 1 illustrates the solution of (23) and the shift in ESS. Note that the impact of $S^{\prime}$ in (23) is magnified by $\left(T_{0}-1\right) ; T_{0}$ is a reproduction-weighted average lifespan (see (24)).

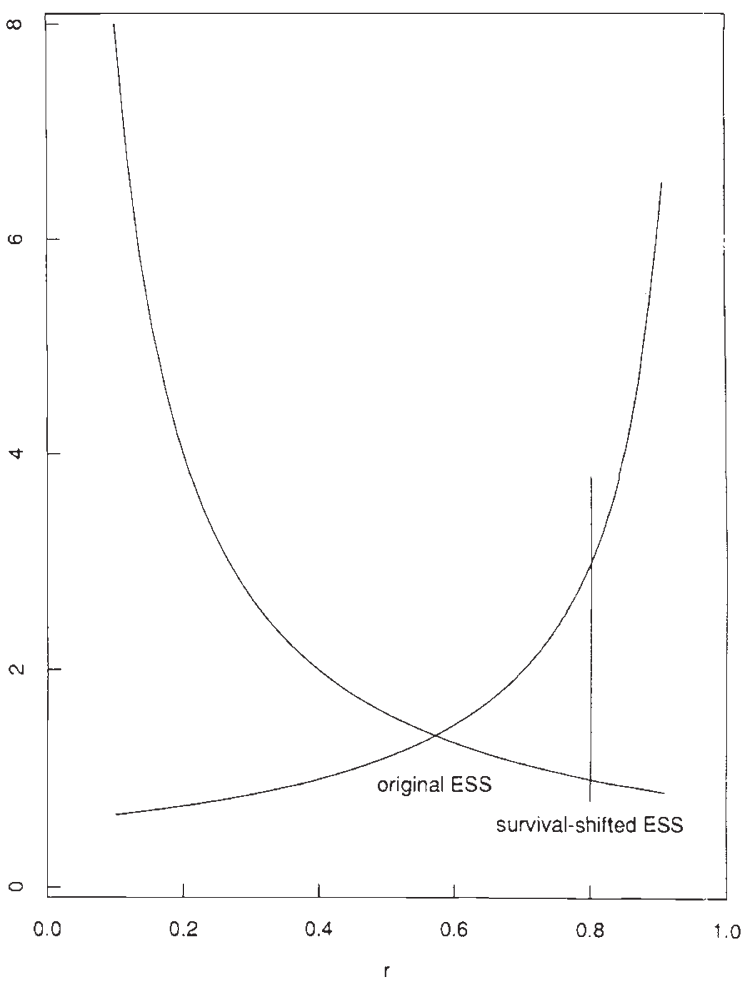

Figure 1 The curves shown intersect at the deterministic ESS. When survival rate increases with allocation, the ESS is shifted to the right as shown. See text equation (22).

\section{THE STOCHASTIC AGE-STRUCTURED ESS}

\section{(i) Structure of the stochastic rule for evolutionary stability}

Focus now on the general stochastic rule. Doing the multiplication in the denominator of (8), using (1) and (7), and using (10), the stochastic rule can be rewritten as

$$
\begin{gathered}
E\left\{\frac { 1 } { ( \overline { F } + \overline { S } ) ( \boldsymbol { V } ^ { T } ( t ) \boldsymbol { U } ( t ) ) } \left[\frac{V_{1}(t) \bar{F}}{2}\left(\frac{\overline{\partial F}}{\bar{F}}+\frac{\overline{\partial M}}{\bar{M}}\right)\right.\right. \\
\left.\left.+\sum_{i=1}^{k-1} V_{i+1}(t) U_{i}(t-1) S_{i}^{\prime}\right]\right\}=0 .
\end{gathered}
$$

The bars are defined as in (11) and

$$
\overline{\partial F}=\overline{\left(\frac{\partial F}{\partial r}\right)}=\sum_{i} \frac{\partial F_{i}(r, t)}{\partial r} U_{i}(t-1) .
$$

The stochastic condition (25) should be compared with the deterministic version (19).

Condition (25) looks much more complicated than Charnov's (1986) product rule which is exemplified by (14). I will first show that (25) 
simplifies to a product rule under certain conditions.

\section{(ii) Simplifying assumptions which yield a product rule}

The most general conditions under which (25) reduces to a product rule are:

(a) survival rates do not vary with allocation;

(b) male and female production functions are separable into shape and level (compare (21));

(c) there is no age-dependence of the shape of the production functions;

(d) there is no randomness in the shape of female production function;

(e) the shape of the male production function varies randomly but is uncorrelated with the female production function.

These conditions imply that

$$
\begin{aligned}
M_{i}(r, t) & =\alpha_{i}(t) Z(t) M(r), \\
F_{i}(r, t) & =\beta_{i}(t) W(t) f(r),
\end{aligned}
$$

where $f$ is deterministic, and $M$ is uncorrelated with the $\beta_{i}$ and $W$. In addition $S_{i}^{\prime}=0$. From (25) and (27) note that

$$
\overline{\partial F}=W(t) \bar{\beta} f^{\prime}, \quad \overline{\partial M}=Z(t) \bar{\alpha} M^{\prime},
$$

so (8) becomes

$$
E\left\{\frac{V_{1}(t) W(t) \bar{\beta} f}{(\bar{\beta} W f+\bar{S})\left(V^{T}(t) U(t)\right)}\left[\frac{f^{\prime}}{f}+\frac{M^{\prime}}{M}\right]\right\}=0 .
$$

Now use assumption (e) above to see that ESS allocation satisfies the product rule

$$
E\left[\frac{f^{\prime}}{f}+\frac{M^{\prime}}{M}\right]=0 .
$$

The simplification (30) is remarkable but depends critically on the assumptions. If either assumption (d) or (e) fails to hold, we cannot factor (30) out of (29). If assumption (a) does not hold, we must add to the left of (29) tems involving $S_{i}^{\prime}$. If assumptions (b) or (c) do not hold, the reduction of $(8)$ to (29) does not go through.

\section{(iii) Fluctuations in female production: beyond the product rule}

The detailed structure of the stochastic rule (8) covers many possibilities. I will consider here only a simple model for an iterocarpic (perennial) plant which illustrates the interplay between fluctuations and demography in determining an ESS. For a population of homozygotes $A A$ which have alloca- tion fraction $r$, I collect all reproducing adults into age-class 2 and write the dynamic equation

$$
\left(\begin{array}{l}
N_{1}(t+1) \\
N_{2}(t+1)
\end{array}\right)=\left(\begin{array}{cc}
0 & F(r, t) \\
s & P(t)
\end{array}\right)\left(\begin{array}{l}
N_{1}(t) \\
N_{2}(t)
\end{array}\right) .
$$

As in the discussion subsequent to equation (1), the age-vector of heterozygotes carrying a rare invading allele $B$ obeys the linearized equation

$$
\left(\begin{array}{c}
\varepsilon_{1}(t+1) \\
\varepsilon(t+1)
\end{array}\right)=\left(\begin{array}{cc}
0 & \Psi\left(r, r_{1}, t\right) \\
s & P(t)
\end{array}\right)\left(\begin{array}{c}
\varepsilon_{1}(t) \\
\varepsilon_{2}(t)
\end{array}\right),
$$

I take male and female production functions to have the form

$$
\begin{gathered}
F(r, t)=W(t) f(r, t) \\
M(r, t)=Z(t) m(r, t),
\end{gathered}
$$

where $f, m$ are randomly varying shape functions. This implies that in (32),

$$
\Psi\left(r, r_{1}, t\right)=\frac{W(t)}{2}\left[f\left(r_{1}, t\right)+\frac{f(r, t)}{m(r, t)} m\left(r_{1}, t\right)\right] .
$$

The general equation (8) is analytically intractable even for this model, so I will concentrate on the case where the fluctuations in $W(t)$ are small in magnitude. I can then use expansion methods (Tuljapurkar, 1982, 1989) to obtain an equation for $a\left(r, r_{1}\right)$, the heterozygote growth rate, and then differentiate as in (5). Details are in the Appendix; the stochastic rule reduces to the condition

$$
\begin{aligned}
& \left(1-\beta \sigma_{p}^{2}-\gamma C_{W F}^{2}\right) E\left(W f \phi^{\prime}\right) \\
& -\nu \operatorname{Cov}\left(W f, W f \phi^{\prime}\right)=0 .
\end{aligned}
$$

Here Cov indicates a covariance; $\beta, \gamma, \nu$ are constants determined by the average demographic rates (see Appendix); $\sigma_{P}^{2}$ is the variance of $P(t)$;

$$
C_{W F}^{2}=\frac{\operatorname{Var}(W f)}{E^{2}(W f)}=\frac{E\left(W^{2} f^{2}\right)-E^{2}(W f)}{E^{2}(W f)} ;
$$

and

$$
\phi^{\prime}=\frac{1}{m(r, t)} \frac{\partial m}{\partial r}+\frac{1}{f(r, t)} \frac{\partial f}{\partial r} .
$$

Recall that the product rule is simply $E\left(\phi^{\prime}\right)=0$. However (35) is quite different because $F$ is correlated with $\phi^{\prime}$ and so averages such as $E\left(W f \phi^{\prime}\right)$ behave quite differently from $E\left(\phi^{\prime}\right)$. This is true even if $W$ and $f$ are uncorrelated. Whenever the female shape function changes randomly over time, the ESS predicted by (35) will not be the same as predicted by a product rule. 
Note that the level of male production, $Z(t)$, does not enter the ESS condition. Thus correlations between $m(r, t)$ and $Z(t)$ have no effect. However, correlations between $m(r, t)$ and either $W(t)$ or $f(r, t)$ have direct effect on (35).

\section{DISCUSSION}

This paper presents a general criterion, equation (8), defining ES sex-allocation for an age-structured population in a fluctuating environment. Three special conditions are required to reduce (8) to a version of Charnov's (1986) product rule. They are:

(a) the shape of the female and male production functions does not change with age;

(b) the shape of the female production function does not vary over time;

(c) fluctuations in the shape of the male production function are statistically independent of all other variation.

Fluctuations in the female shape function complicate the ESS criterion considerably. Correlations between the male shape function and male level of production are irrelevant to the ESS. But correlations between all other components of production do matter.

When survival rates change with sex-allocation, the ESS shifts in the direction expected from a consideration of the relative costs of the two sexes. The impact of survival rates at a particular age depends also on the fraction of lifetime reproduction which occurs up to that age.

Interesting open issues remain: the effect of cyclical variation in production; the possibility that the sex-allocation fraction is age-dependent; whether the stochastic rule (8) derived here allows multiple ESS's; and, the enormous range of stochastic genetic questions which complement those explored by Karlin and Lessard (1986).

\section{APPENDIX}

\section{(i) Deriving (8)}

The vectors defined in (6) are described fully by Hajnal (1976), Tuljapurkar (1982, 1989). The sequences $\boldsymbol{V}(t), \boldsymbol{U}(t)$ satisfy the recursions

$$
\boldsymbol{U}(t)=\frac{\boldsymbol{X}(t) \boldsymbol{U}(t-1)}{\lambda(t)}, \quad \boldsymbol{V}^{T}(t-1)=\frac{\boldsymbol{V}^{T}(t) \boldsymbol{X}(t)}{\eta(t)},
$$

where $\lambda(t)$ is defined in (7) and

$$
\eta(t)=\sum_{i}\left(\boldsymbol{V}^{T}(t) \boldsymbol{X}(t)\right)_{i} .
$$

The stochastic growth rate is defined as

$$
\begin{aligned}
a & =\lim _{n \rightarrow \infty} a_{n}, \\
a_{n} & =\frac{1}{n} \log \|\boldsymbol{X}(n) \boldsymbol{X}(n-1) \cdots \boldsymbol{X}(1)\| \\
& =\frac{1}{n} \log \left[\boldsymbol{V}^{T}(n) \boldsymbol{X}(n) \boldsymbol{X}(n-1) \cdots \boldsymbol{X}(1) \boldsymbol{U}(0)\right] .
\end{aligned}
$$

If the $\boldsymbol{X}(t)$ depends on an argument $r$, the derivative $(\partial a / \partial r)$ is computed by replacing $\boldsymbol{X}(t)$ in (A2) by $\boldsymbol{X}(t)+\eta \boldsymbol{X}^{\prime}(t)$ where $\boldsymbol{X}^{\prime}=(\partial \boldsymbol{X} / \partial r)$, so that $a_{n}$ changes to $a_{n}+\eta b_{n}$. Then $(\partial a / \partial r)$ equals $\operatorname{Lim}\left(b_{n}\right)$ as $n \rightarrow \infty$. Copying the methods in Tuljapurkar (1982, Appendix),

$$
b_{n}=\frac{1}{n} \sum_{j=1}^{n} \frac{\boldsymbol{V}^{T}(j) \boldsymbol{X}^{\prime}(j) \boldsymbol{U}(j-1)}{\left.\lambda(j) \boldsymbol{V}^{T}(j) \boldsymbol{U}(j)\right)},
$$

and

$$
\lim _{n \rightarrow \infty} b_{n}=E\left\{\frac{\boldsymbol{V}^{T}(t) \boldsymbol{X}^{\prime}(t) \boldsymbol{U}(t-1)}{\lambda(t)\left(\boldsymbol{V}^{T}(t) \boldsymbol{U}(t)\right)}\right\} .
$$

In this paper the matrix sequence we differentiate is actually the one in equation (3) of the text. However, to complete the calculation we must set $r_{1}=r$ in accordance with (5). Therefore (A3) gives the correct value of $\left(\partial a_{1} / \partial r_{1}\right)$ when $r_{1}=r$, with $\boldsymbol{X}^{\prime}(t)$ replaced by the matrix of equation (9).

\section{(ii) Stochastic growth rate for (32)}

The method used is derived in Tuljapurkar (1982); see Lande (1987) for a different application. Start with the average of the matrix in (32), given by

$$
\left(\begin{array}{ll}
0 & \psi \\
s & p
\end{array}\right), \quad \text { where } \psi=E \Psi, p=E P
$$

This has

(a) dominant eigenvalue $\lambda=(p / 2)+\frac{1}{2} \sqrt{p^{2}+s \psi}$;

(b) corresponding right eigenvector $U^{T}=$ $(1, \lambda / \psi)$

(c) corresponding left eigenvector $V^{T}=(1, \lambda / s)$. Let $T=V^{T} U$. The formula in Tuljapurkar (1982) asserts that for small fluctuations in $\Psi$ and $P$ the stochastic growth rate of the heterozygote is

$$
a\left(r_{1}, r\right)=\log \lambda-\frac{1}{2 \lambda^{2} T^{2}}\left[U_{2}^{2} \sigma_{\psi}^{2}+V_{2}^{2} U_{2}^{2} \sigma_{P}^{2}\right],
$$


where $\sigma_{\psi}^{2}$ is the variance of $\Psi$ and $\sigma_{P}^{2}$ the variance of $P$. To compute $\left(\partial a / \partial r_{1}\right)$, use the characteristic polynomial of the average matrix to find that

$$
\frac{\partial \lambda}{\partial r_{1}}=\frac{s \psi^{\prime}}{T(\lambda-p)}, \quad \text { with } \psi^{\prime}=\frac{\partial \psi}{\partial r_{1}} \text {. }
$$

Note that when $r_{1}=r$, equation (34) shows that

$$
\begin{aligned}
E \psi & =E(W f), \\
E \psi^{\prime} & =\frac{1}{2} E\left(W f \phi^{\prime}\right) \quad \text { where } \phi^{\prime} \text { is given by (37), } \\
\left.\frac{\partial E \psi^{2}}{\partial r_{1}}\right|_{r_{1}=r} & =E\left(W^{2} f^{2} \phi^{\prime}\right) .
\end{aligned}
$$

Using these in (A4) one finds that

$$
\begin{aligned}
\left(\frac{2 \lambda(\lambda-p) T}{s}\right)\left(\left.\frac{\partial a}{\partial r_{1}}\right|_{r_{1}=r}\right) & \\
= & E\left(W f \phi^{\prime}\right)\left[1-\frac{\lambda \sigma_{p}^{2}\{p(\lambda-p)-T\}}{T^{3}(\lambda-p)^{3}}\right] \\
& +E\left(W f \phi^{\prime}\right) C_{W F}^{2}\left[\frac{\lambda(\lambda-p)}{s T E(W f)}-\frac{\lambda p}{T^{3}(\lambda-p)^{2}}\right] \\
& -\frac{\lambda(\lambda-p)}{s T E^{2}(W f)} \operatorname{Cov}\left(W f, W f \phi^{\prime}\right) .
\end{aligned}
$$

This leads to (35) with $\beta \gamma, \nu$ directly identifiable.

Acknowledgements I thank NIH for support under HD-16640 and HD-00693; also Portland State University. This analysis was stimulated by discussions in Marc Feldman's group at Stanford-G. Gandini, A. M. John, M. Twomey, P. Wiener.

\section{REFERENCES}

BIERZYCHUDEK, P. 1984. Assessing “optimal" life histories in a fluctuating environment: The evolution of sex-changing by jack-in-the-pulpit. Am. Nat., 123, 829-840.

BULL, J. J. 1981. Sex ratio evolution when fitness varies. Heredity, 46, 9-26.

CASWELL, H. 1978. A general formula for the sensitivity of population growth rate to changes in life history parameters. Theor. Pop. Biol., 14, 215-230.

CHARLESWORTH, B. 1980. Evolution in Age-Structured Populations. Cambridge Univ. Press, Cambridge.

CHARNOV, E. L. 1979. The genetical evolution of patterns of sexuality: Darwinian fitness. Am. Nat., 113, 465-480.

CharnOV, E. L. 1982. The Theory of Sex Allocation. Princeton Univ. Press, Princeton.

CHARNOV, E. L. 1986. An optimization principle for sex allocation in a temporally varying environment. Heredity, 56, 119-121.

CHARNOV, E. L. 1988. Hermaphroditic sex allocation with overlapping generations. Theor. Pop. Biol., 34, 38-46.

COHEN, J. E. 1979. Comparative statics and stochastic dynamics of age-structured populations. Theor. Pop. Biol., 17, 159171.

HAJNAL, L. 1976. On products of non-negative matrices. Math. Proc. Camb. Phil. Soc., 79, 521-530.

HEYDE, C. C. AND COHEN, J. E. 1985. Confidence intervals for demographic projections based on products of random matrices. Theor. Pop. Biol., 27, 120-153.

KARLIN, S. AND S. LESSARD. 1986. Theoretical Studies on Sex Ratio Evolution. Princeton Univ. Press, Princeton.

LANDE, R. 1987. Extinction thresholds in demographic models of territorial populations. Am. Nat., 130, 624-635.

MAYNARD SMITH, J. 1982. Evolution and the Theory of Games. Cambridge Univ. Press, Cambridge.

SEGER, J. 1983. Partial bivoltinism may cause altering sex-ratio biases that favor ensociality. Nature, 301, 59-62.

TULJAPURKAR, S. D. 1982. Population dynamics in variable environments. III. Evolutionary dynamics of r-selection. Theor. Pop. Biol., 21, 141-165.

TULJAPURKAR, S. D. 1989. An Uncertain Life: Demography in random environments. Theor. Pop. Biol., 35 (In Press).

TUlJapurkar, S. D. AND ORZACK, S. H. 1980. Population dynamics in variable environments. I. Long-run growth rates and extinction. Theor. Pop. Biol, 18, 314-342.

WERREN, J. H. AND CHARNOV, E. L. 1978. Facultative sexratio and population dynamics. Nature, 272, 349-350. 